Lindblad: I should like to ask if an alternative to the spiral structure has been contemplated, for instance, a sort of elliptical ring with apsidal line inclined towards the direction of the Sun.

Oort: One difficulty would be that some of the very prominent features such as the high positive velocity at negative longitudes are not consistent with such an analysis.

Perek: Is it possible to assume that the two spiral arms are symmetrical or is there any indication of asymmetry?

Oort: There seems to be a higher expansion velocity in the matter behind the centre than in the arm nearest to us.

Aller: Do the data exclude the existence of a bar at the centre of the Galaxy?

Oort: No, but the structure cannot be symmetrical on both sides; if a bar is present, it would have to be asymmetrical.

\title{
43. HYDROGEN MOTIONS IN THE CENTRAL REGION OF THE GALAXY
}

\section{B. F. Burke and M. A. Tuve}

Carnegie Institution of Washington, Department of Terrestrial Magnetism

The 21-cm hydrogen radiation near the galactic plane exhibits strong deviation from circular motion over longitudes within $30^{\circ}$ or so of the galactic centre. In order to extend the observations first made by Rougoor and Oort,* the Carnegie multichannel $\mathrm{H}$-line spectrograph was used with our 60 -foot radio telescope at Derwood, Maryland, in order to confirm the Leiden work and extend observations to more southern longitudes. Cross-sections extending $\pm 2^{\circ}$ in latitude were taken every $2^{\circ}$ in longitude from $l \mathrm{II}=-26^{\circ} .8$ to $l \mathrm{II}=+2^{\circ} 2$ and a series of points along the galactic equator were taken every $1^{\circ}$ over the same range. Effort has been concentrated on the southern extension of the observations, which agree well with the Leiden observations over the section in common, but which carry the observations nearly $20^{\circ}$ farther south in longitude.

The observations in the plane are summarized in Figures 1 and 2, which show, respectively, the run of the hydrogen maxima and the temperature contours as a function of $l^{\mathrm{I}}$ and $V_{r}$. The " $3 \cdot 5$-kpc expanding arm" dominates the diagrams from the centre to about $l^{\mathrm{I}}=320^{\circ}\left(l^{\mathrm{II}}=-7 \cdot 8\right)$, but farther south the line profiles are much more complicated, showing three or four peaks as a general rule. The " $3 \cdot 5$ kpc arm" appears, in Figure 1, to break into two parts at $l \mathrm{I}=320^{\circ}$, although the possibility exists that two distinct arms are being observed that just happen to merge in velocity at this point. At $l \mathrm{I}=315^{\circ}$, the " $3 \cdot 5$ - $\mathrm{kpc}$ arm" also appears to undergo a change, and examination of the records indicates that there is still a weak extension (not shown in Figure 1) to about $l^{\mathrm{I}}=312^{\circ}$ of a feature whose velocity varies linearly with $l$. The weakest contour line in Figure 2 shows the remnant clearly. Figure 3 shows a series of cross-sections in $b$ that also show the highvelocity extension, which no longer lies in the plane at $l^{\mathrm{I}}=312^{\circ}\left(l^{\mathrm{II}}=345^{\circ} \cdot 3\right)$ but is clearly defined, with a velocity of nearly $160 \mathrm{~km} / \mathrm{sec}$.

*Rougoor, G. W., and Oort, J. H. (1960).-Proc. Nat. Acad. Sci. (Washington) 46: 1-13. 
A second clearly distinguishable feature, first visible at $l \mathrm{I}=323^{\circ}$ and clearly visible from $l^{\mathrm{I}}=320^{\circ}$ south, shows the same behaviour as the $3 \cdot 5$-kpc arm, with a linear variation that implies a weak expansion of about $15 \mathrm{~km} / \mathrm{sec}$, and a slope that gives a rotation that has about the same $\omega(R)$ as does the " $3 \cdot 5-\mathrm{kpc}$ arm". The slope changes at $l^{\mathrm{I}}=316^{\circ}$, from which point there is some ambiguity with respect to the first branch of the " $3 \cdot 5-\mathrm{kpc}$ arm".

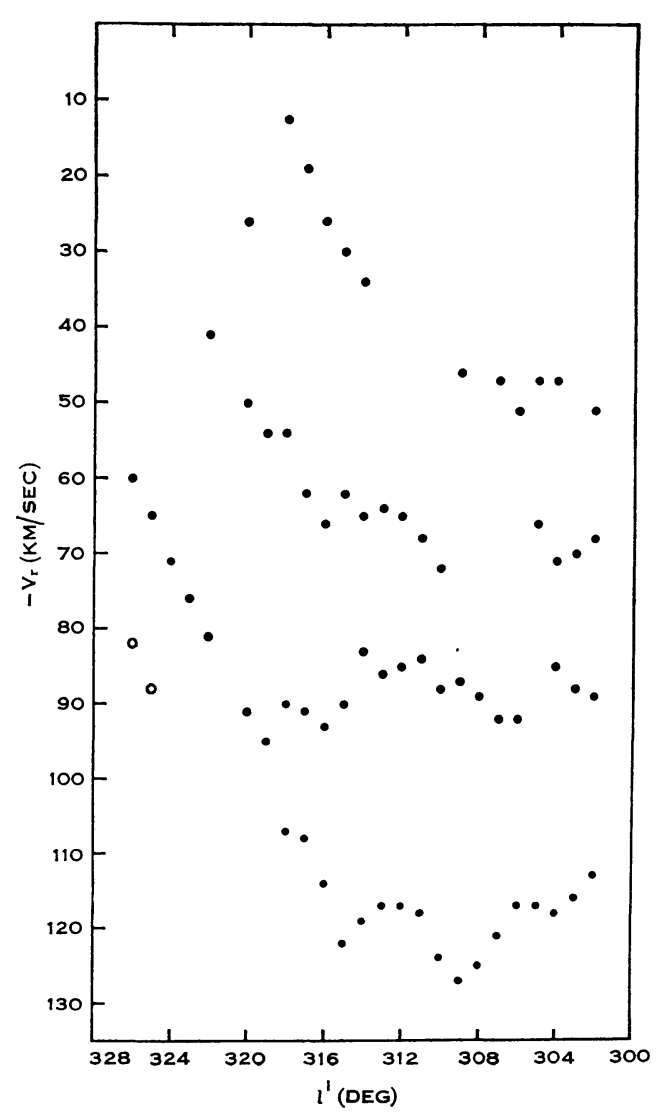

Fig. 1.-Observed velocities of hydrogen maxima along galactic plane near galactic centre. The " $3 \cdot 5$-kpc arm" is the feature having the most negative velocities.

A third feature can be seen at the top of the diagram, whose slope and $V_{r-}$ intercept are consistent with an extension of the $3 \cdot 5$ - $\mathrm{kpc}$ arm on the far side of the galactic centre.

Interpretation of the details of the observed motions is difficult, and complicated by the fact that the angular sizes of the features, as seen in latitude profiles such as Figure 3, do not vary in a sufficiently regular way to give confidence in the use of angular extent as a distance indicator. An attempt has been made to fit simple models 
to the observations, and to interpret the deviations. An example is shown in Figure 2, where model expanding arms were fitted to the observed contours. If the features observed were simple circular arms, uniformly rotating and expanding, the para-

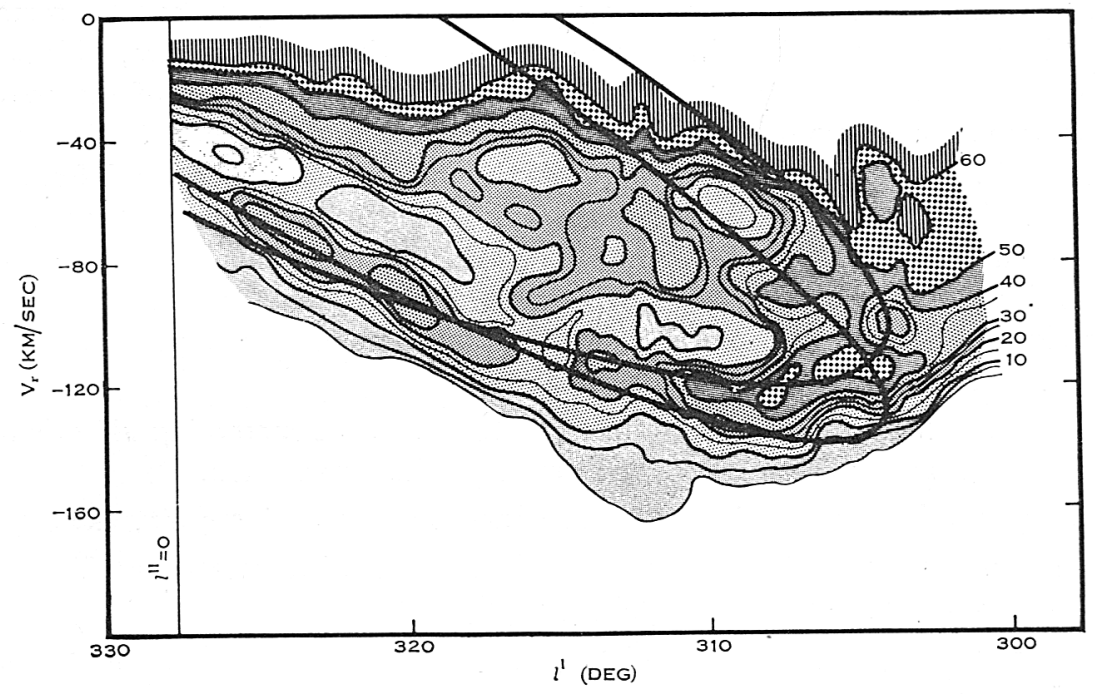

Fig. 2.-Hydrogen intensity in ${ }^{\circ} \mathrm{K}$ uncorrected for antenna resolving pattern, observed along galactic plane as function of radial velocity and longitude.

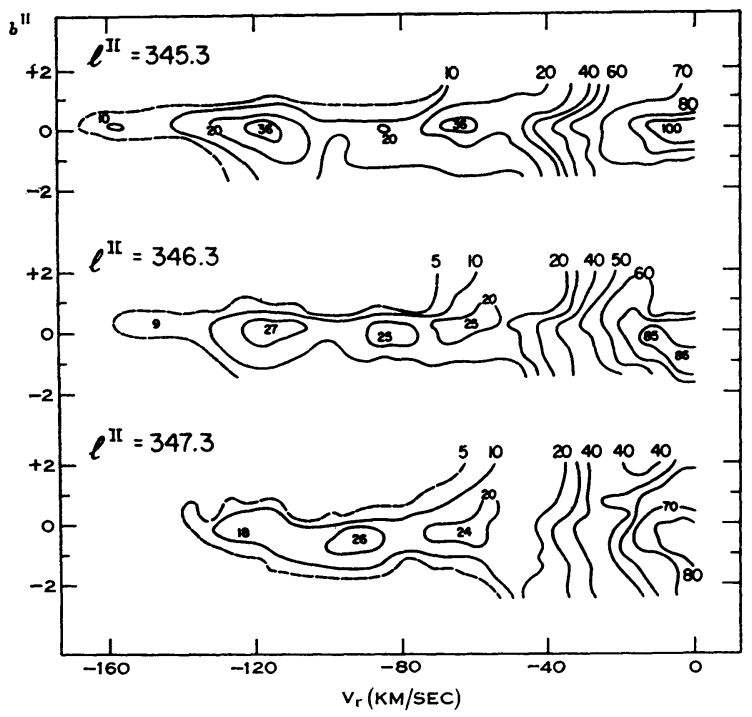

Fig. 3. - Sample cross-sections in latitude showing expanding features in the inner parts of the Galaxy. Antenna temperature is given in ${ }^{\circ} \mathrm{K}$, referred to $l \mathrm{I}=50^{\circ}$, $b^{\mathbf{I}}=0^{\circ}$, assumed to be $100^{\circ}$ peak.

meters that are of interest are the expansion velocity $\tilde{\omega}$, the circular velocity $\omega$, and the distance from the centre $R$. At the longitude of the centre, the expansion velocity can be determined from the $V_{r}$-intercept and the product $R_{0}\left[\omega(R)-\omega\left(R_{0}\right)\right]$ 
can be measured from the slope, $\mathrm{d} V_{r} / \mathrm{d} l$. At the tangent point, the coordinates of the turnaround give $R$ (if $R_{0}$ is known), and $R_{0}\left[\omega(R)-\omega\left(R_{0}\right)\right]$, while the rate of opening of the curve gives $\tilde{\omega}$.

Such a simple model does not fit our observations well, as can be seen in Figure 2. Two models were tried in fitting the major hydrogen maximum, with parameters chosen in the first to fit the observations near the longitude of the centre, and in the second model to fit the observations near the tangent point (which is not clearly defined). Neither model fits the observations at the opposite end from the fitting, and it is evident that the " $3 \cdot 5-\mathrm{kpc}$ arm" is not a simple circular feature. The sign of the deviations is interesting, however, because if one follows a model curve that fits near $l \mathrm{II}=0^{\circ}$, the observed hydrogen velocities are systematically too large for the highest-velocity feature. Such a variation can be explained by the following models:

(1) A spiral feature, with $\Delta \omega$ and $\tilde{\omega}$ decreasing with increasing $R$, oriented as an opening spiral, leading rather than trailing galactic rotation.

(2) A spiral feature, trailing, with $\Delta \omega$ increasing with increasing $R$.

(3) A spiral feature, trailing, with $\omega$ increasing with increasing $R$.

Of course a combination of (2) and (3) is also possible.

Model (1) is possible but unlikely, since no such structures have been observed in external galaxies. Model (2) also appears unlikely, although so little is known of galactic dynamics that it remains a possibility. Model (3) also is not the kind of steady motion one would ordinarily expect, but if the expansion motions are the results of an explosion of cataclysmic nature in the galactic nucleus, it does not seem altogether unlikely, and may be taken as evidence for such events giving rise to the observed outflow. One advantage of such a model is that the loss of mass from the centre, which is impossibly high in a steady-state model, is no longer as serious, since we are now seeing the peak and not the average outflow. A further suggestive circumstance is the apparent lack of hydrogen within $3 \mathrm{kpc}$. From $l^{\mathrm{II}}=0$ to about $l^{\mathrm{II}}=-13: 8$ $\left(l^{\mathrm{I}}=314^{\circ}\right)$ there seem to be no tangent spiral features, implying that the inner $2 \mathrm{kpc}$ is nearly empty, although there may be a tangent arm at about $l^{\mathrm{I}}=314^{\circ}$. The major feature, the "3.5-kpc arm", may well represent the hydrogen swept up by an explosion in the nucleus, still expanding, though decelerated, and apparently branching at two points at least. Although the evidence seems to support the explosion hypothesis, it should be emphasized that serious difficulties remain in the interpretation of the remaining features, particularly on the northern side of the galactic centre.

\section{Discussion}

de Vaucouleurs: How definite is the evidence for the branching off of the three arms (as drawn on the board) from a single arm, as against three separate lanes crossing the line of sight to the centre at several distances?

Burke: We are unable to trace the secondary features past the point at which they merge with the principal expanding feature. 